\title{
Gute ärztliche Beratung ist zentral für die HIV-Prävention!
}

\author{
Kommentar des Bundesamts für Gesundheit und der Eidg. Kommission für Aids-Fragen EKAF zum Artikel Meystre-Agustoni
}

Bundesamt für Gesundheit, Abteilung Übertragbare Krankheiten, Sektion Aids

HIV-Prävention in der Schweiz basiert auf den drei Interventionsebenen der bevölkerungsbezogenen Information (STOP-AIDS-Kampagne), der zielgruppenspezifischen Information und Motivation sowie der individuellen Beratung. Die individuelle Beratung findet am häufigsten in der Arztpraxis statt; dieses Potential wird jedoch zuwenig systematisch genutzt. Die Fachkommission Klinik und Therapie HIV/Aids FKT hat zur Unterstützung der Ärztinnen und Ärzte Empfehlungen zur HIV-Testberatung («Vademecum» in dieser Ausgabe) erarbeitet.

a Der Artikel betrachtet die Beratung von Menschen mit negativem oder unbekanntem Serostatus; die präventive Beratung von HIV-positiven Menschen wird hier nicht thematisiert, ist aber ein Bereich, den das BAG im Nationalen HIV/Aids-Programm 2004-2008 weiterentwickeln wird.

b Gemäss Schweizerischer Gesundheitsbefragung und Zahlen des BAG.

c Siehe dazu u.a. folgende Publikationen: [2-4].
Der vorliegende Artikel von Meystre-Agustoni et al. befasst sich mit der Risikoanamnese und Beratung zu HIV in Schweizer Arztpraxena. Er zeigt, dass sich diese seit 1990 auf einem relativ hohen Niveau bewegen, dabei aber Mängel aufweisen.

Die HIV-Risikoanamnese wird erfreulicherweise zunehmend über die klassischen Risikogruppen hinaus durchgeführt. In einzelnen Gruppen, z.B. bei Migrantinnen und Migranten, wird HIV jedoch nach wie vor zuwenig thematisiert, während die Thematisierung in der «klassischen» Risikogruppe der homosexuellen Männer gar abnahm.

Individuelle Präventionsberatung kann unabhängig von der Durchführung eines HIV-Tests sinnvoll und wichtig sein, beispielsweise bei Unklarheiten bezüglich der Safer-Sex-Regeln oder zur Integration der Prävention in die persönliche Lebenssituation. Häufig ist die Präventionsberatung jedoch mit der Durchführung eines HIV-Tests verbunden, der von der Schweizer Bevölkerung breit beansprucht wird: Gemäss einer neuen Studie [1] haben sich 41\% eines Zufallssamples von Personen zwischen 18 und 45 Jahren ausserhalb des Blutspendens mindestens einmal im Leben auf HIV testen lassen. Jährlich werden in der Schweiz rund 300000 HIV-Tests ausserhalb des Blutspendens durchgeführt $^{\mathrm{b}}$. Die individuelle Beratung im Rahmen des HIV-Tests stellt somit ein enormes Potential der HIV-Prävention dar.

Knapp die Hälfte (48\%) der HIV-Tests mit positivem Resultat in der Schweiz werden in der ärztlichen Praxis durchgeführt. Ärztinnen und
Ärzte sind somit wichtige Träger der individuellen Beratung zu HIV/Aids in der Schweiz! Gemäss der vorliegenden Studie anerkennen die Ärztinnen und Ärzte ihre wichtige Rolle.

HIV-Testberatung wird unter dem Stichwort VCT (Voluntary Counseling and Testing - Beratung und Testen auf freiwilliger Basis) diskutiert. VCT wurde Mitte der achtziger Jahre von UNAIDS als Standardprozedur für alle Personen entwickelt, die ihren Infektionsstatus kennenlernen und einen HIV-Test machen wollten. Die Kernelemente sind in den Empfehlungen verschiedener Gesundheitsorganisationen festgehaltenc. Seitens der USA wird seit einiger Zeit eine breite HIV-Testung gefördert, bei der aber nicht immer klar ist, inwiefern die Beratung gewährleistet ist. Dies gilt insbesondere für den in den USA nun zugelassenen HIV-Speicheltest. Vor diesem Hintergrund erhält die Sicherung der Beratungsqualität neue Aktualität.

Dass das Beratungspotential im Rahmen des HIV-Tests in der Schweiz nur teilweise genutzt wird, zeigt der vorliegende Artikel, aber auch Studien unter den Nutzern des Angebots: 44\% der homosexuellen Männer beispielsweise, die im Jahr 2002 einen Test machen liessen, erhielten weder vor noch nach dem Test eine Beratung [5]. Gemäss der vorliegenden Studie sprechen ein Drittel der Ärztinnen und Ärzte bei einer Testberatung die Frist zwischen HIV-Exposition und Nachweisbarkeit des Virus nicht systematisch an, und über die Hälfte von ihnen nehmen nicht systematisch eine Risikoabklärung vor. Dadurch kann der Test als präventives Instrument nicht voll genutzt und auch die Sicherheit des Resultats nicht umfassend gewährleistet werden.

Die Befunde von Meystre-Agustoni et al. werden durch eine soeben veröffentlichte linguistische Studie bei niedergelassenen Ärztinnen und Ärzten in der Romandie ergänzt [6]: Eine Mehrzahl der 620 befragten Grundversorger gibt an, dass ihnen der übliche medizinische Jargon eher im Weg steht, um ihre Patientinnen/Patienten auf die Themen Sexualität und HIV/Aids 
anzusprechen. Auch besteht eine gewisse Befangenheit beiderseits und auf medizinischer Seite oft ein Mangel an Zeit für eine HIV-Beratung. Knapp die Hälfte betonen die Wichtigkeit einer guten Zusatzausbildung in nonverbaler Kommunikation und in der eigentlichen Gesprächsund Beratungstechnik.

BAG und EKAF sind sich bewusst, dass die Unterstützung der Ärztinnen und Ärzte für eine qualitativ optimale HIV-Anamnese und -Beratung noch verbessert werden kann. Zwar existieren umfassende Informationen zu Test und
Diagnostik zuhanden der Ärztinnen und Ärzte [7] sowie Empfehlungen zur HIV-Testung in der Schwangerschaft [8] und ein revidiertes «HIVTestkonzept 2004» [9]. Aber es fehlt ein kurzes Merkblatt zur Risikoanamnese, Beratung und Testung zu HIV. In den Bereichen Labor, Diagnostik und Therapie von HIV/Aids sind solche Empfehlungen längst etablierte Instrumente zur Unterstützung des medizinischen Personals. Die in dieser Ausgabe publizierten Empfehlungen der FKT zur HIV-Testberatung («Vademecum») schliessen diese Lücke.

\section{Präventionsbotschaften}

(gemäss Nationalem HIV/Aids-Programm 2004-2008, S. 25)

- Jede Person übernimmt im Rahmen ihrer Möglichkeiten selbst Verantwortung, sich vor einer HIV-Infektion zu schützen.

- Wer sexuell aktiv ist und nicht in einer gegenseitig treuen und HIV-freien Beziehung lebt, soll die Regeln des Safer Sex befolgen:

- bei eindringendem Verkehr immer ein Präservativ guter Qualität verwenden;

- kein Sperma oder Menstruationsblut in den Mund nehmen oder schlucken.

- Wer sexuell aktiv ist und nicht in einer gegenseitig treuen und HIV-freien Beziehung lebt, soll sich zudem über Risiken und Schutzmöglichkeiten informieren und den Gebrauch des Präservativs üben.

- In einer gegenseitig treuen Beziehung soll erst auf das Präservativ verzichtet werden, wenn beide Partner frühestens nach drei Monaten (gemeinsam) beraten wurden und einen negativen HIV-Test haben.

- Ein HIV-Test schützt nicht vor Aids. Wer jedoch annimmt, sich riskant verhalten zu haben, kann sich mit einem Test Klarheit verschaffen und ermöglicht damit u.U. eine frühzeitige Behandlung der HIV-Infektion.

- Wer intravenös Drogen konsumiert, soll immer eine neue Spritze und sauberes eigenes Besteck (Löffel, Filter, Watte, Wasser) verwenden und dieses nicht mit anderen Konsumentinnen/Konsumenten teilen (Safer Use).

- HIV und Aids sind behandelbar, aber nicht heilbar. Die Behandlung ist aufwendig, beschwerlich, teuer und in ihrem Erfolg nicht gewiss. Das Präservativ ist der beste Schutz vor HIV/Aids.

- HIV/Aids ist nach wie vor ein gesellschaftliches Problem. Unterstützung und Solidarität gegenüber Betroffenen und gefährdeten Personen ist eine Notwendigkeit.

- HIV-Prävention hat Grenzen. Verständnis und Offenheit gegenüber Menschen mit HIV/Aids gehören zu einer solidarischen Gesellschaft und stärken wiederum die HIV-Prävention.

- HIV wird nicht übertragen beim Küssen, bei sozialen Kontakten wie Händedruck, Umarmen und Streicheln, durch Niesen oder Husten, beim gemeinsamen Essen und Trinken und Benutzen derselben Teller, Gläser und Bestecke, beim Zusammenarbeiten und -wohnen mit Menschen mit HIV/Aids, beim Benutzen von Bädern, Toiletten oder Saunen und auch nicht über Insektenstiche.

\section{Literatur}

1 Renzi C, Zantedeschi E, Signorelli C, NEM Group. Voluntary HIV testing in Europe. Scand J Public Health 2004;32:102-10.

2 WHO. Increasing access to knowledge of HIV status: conclusions of a WHO consultation, 3-4 December 2001. Geneva: WHO, 2002.

3 UNAIDS. Voluntary Counselling and Testing: a gateway to prevention and care. UNAIDS Case study. Geneva: UNAIDS; 2002.
4 CDC. Revised Guidelines for HIV Counseling, Testing, and Referral (CTR) and Revised Recommendations for HIV Screening of Pregnant Women. MMWR 2001;50:1-58.

5 Häusermann M, Wang J. projet santé gaie. Les premiers résultats de l'enquête sur la santé des hommes gais de Genève. Genève: Dialogai; 2003. www.dialogai.org/pdfs/brochurecomplet.pdf. 
6 Singy P. Le sida au cabinet médical. Les mots pour en parler. Genève: Editions Médecine et Hygiène; 2004.

7 Bundesamt für Gesundheit. HIV - aktuelles Wissen zu Test und Diagnostik. Informationen für Ärztinnen und Ärzte. Bern: Bundesamt für Gesundheit in Zusammenarbeit mit EKAF; 2000. www.bag.admin.ch/aids/publikationen/d/aids.pdf.

8 Fachkommission Klinik und Therapie HIV/Aids. HIV-Testung während der Schwangerschaft. Empfehlung der Fachkommission Klinik und Therapie HIV/Aids. Bulletin des Bundesamtes für Gesundheit 2003;9:152-3. www.suchtundaids. bag.admin.ch/imperia/md/content/aids/18.pdf.
9 Fachkommission Labor und Diagnostik. Diagnostische Empfehlung. HIV-Testkonzept 2004: Neue Richtlinien zum Screening. Bulletin des Bundesamtes für Gesundheit 2004;10:168-70. www.hiv.ch/rubriken/epidx/diagnose/ testkonzeptd_2004.pdf.

\section{HIV-Testberatung: ein kurzes Vademecum für die Praxis}

Pietro Vernazza, Fachkommission Klinik und Therapie HIV/Aids

In der Schweiz werden in Arztpraxen und Spitälern jährlich etwa 300000 HIV-Testungen durchgeführt. Jede HIV-Testung soll von einem kurzen Testgespräch begleitet werden. Dieses kurze Vademecum fasst die wichtigsten Punkte zum Testgespräch zusammen.

\section{Grundsätze für die HIV-Testung}

- Personen, die sich wiederholt auf das Vorliegen einer HIV-Infektion testen lassen, gehen meist ein wiederholtes Risiko ein.

- Jeder HIV-Test bietet eine Gelegenheit für ein kurzes Präventionsgespräch.

- Ein HIV-Test ohne Testberatung ist eine verpasste Gelegenheit zur HIV-Prävention.

- Präventionsarbeit gehört zu den wichtigsten Aufgaben der Grundversorger.

\section{Mögliche Einstiegsfragen für das Testgespräch}

\section{Fragen zum Risikoverhalten}

- Wann hätten Sie sich zuletzt mit HIV infizieren können?

- Was macht Sie so sicher, dass es danach nicht hätte sein können?
- Wie lange dauert es Ihrer Meinung nach, bis ein Test positiv ausfallen kann?

- Hatten Sie seit dem Risikokontakt Fieber, einen Hautausschlag am Körper oder andere Krankheitszeichen?

\section{Fragen zum Präventionsverhalten}

- Was wissen Sie über mögliche vorbeugende Massnahmen?

- Wo sehen Sie die grössten Schwierigkeiten, wenn es um die HIV-Prävention geht?

- Ist Ihrer Meinung nach HIV-Prävention wichtiger als die Prävention von anderen Geschlechtskrankheiten?

- Hatten Sie schon einmal Probleme beim Verwenden eines Kondoms?

- Wissen Sie, wie man Kondome gebraucht? Könnten Sie es jemand anderem erklären?

\section{Das Testresultat ist negativ: häufige Fehlinterpretationen}

- Dann kann ich sicher sein, dass der Partner meines letzten Sexualkontakts negativ ist, auch wenn ich seinen Status nicht kenne:

$\rightarrow$ Falsch: Es hat bisher einfach noch nicht zu einer Infektion geführt. 
- Dann war mein Verhalten bisher o.k., ich kann so weitermachen:

$\rightarrow$ Falsch: Das bisherige Verhalten kann durchaus ein hohes Risiko beinhalten. Es hat bisher einfach noch nicht zu einer Infektion geführt.

- Dann habe ich letzte Woche Glück gehabt: $\rightarrow$ Falsch: Dass es bei dieser Risikoexposition letzte Woche nicht zur Infektion kam, kann frühestens in drei Monaten mit Sicherheit ausgeschlossen werden.

- Dann muss ich jetzt meinem Partner nichts sagen:

$\rightarrow$ Falsch: Da der Zeitpunkt der Risikoexposition noch nicht drei Monate zurückliegt, kann eine Infektion noch nicht sicher ausgeschlossen werden. Der Partner/die Partnerin kann also nach wie vor gefährdet sein.

- Dann sind meine aktuellen Krankheitssymptome nicht auf eine HIV-Infektion zurückzuführen:

$\rightarrow$ Achtung: Bei Verdacht auf eine HIVPrimoinfektion muss unbedingt das HIVAntigen bestimmt werden (als einzelner Test), noch besser ist eine wiederholte Bestimmung von Antigen und Antikörpern in 5-8 Tagen (Kombo-Test).
Wichtige Informationen vor der Durchführung eines Schnelltests

- Der HIV-Schnelltest kann keine akute Infektion (kein HIV-Antigen!) entdecken. Ein kombinierter HIV-Antikörper/Antigen-Test (4. Generation) eignet sich besser.

- Ein HIV-Schnelltest ist ein Suchtest. Er kann eine HIV-Infektion frühestens nach drei Monaten wirksam ausschliessen. Ein reaktives Testresultat kann durchaus falsch positiv sein (etwa 1mal auf 1000 negative Testresultate). Bei tiefer Prätestwahrscheinlichkeit kann der falsch positive Voraussagewert bei $50-70 \%$ liegen. Für die Bestätigung des Resultates bzw. für den Ausschluss einer Infektion muss Blut in ein Labor gesandt werden. Es empfiehlt sich, die zu testenden Personen vor der Durchführung des Schnelltests darauf aufmerksam zu machen.

\section{Literatur}

- Bundesamt für Gesundheit. HIV - aktuelles Wissen zu Test und Diagnostik. Informationen für Ärztinnen und Ärzte. Bern: Bundesamt für Gesundheit in Zusammenarbeit mit EKAF; 2000. www.bag.admin.ch/aids/publikationen/d/aids.pdf.

- Fachkommission Labor und Diagnostik. Diagnostische Empfehlung. HIV-Testkonzept 2004: Neue Richtlinien zum Screening. Bulletin des Bundesamtes für Gesundheit 2004;10:168-70. www.hiv.ch/rubriken/epidx/diagnose/ testkonzeptd_2004.pdf. 